\title{
Proposed Criteria Positions PSMA PET for the Future
}

\author{
Steve Y. Cho \\ University of Wisconsin School of Medicine and Public Health, and Carbone Comprehensive Cancer Center, Madison, Wisconsin
}

See the associated articles on pages 469 and 479.

Kairos (кацро́) —an Ancient Greek word meaning the right, critical or opportune moment; a proper or opportune time for action. ${ }^{(I)}$

\section{$\mathbf{P}$} rostate cancer is an oncologic disease that has been deprived of the benefits of molecular imaging in this era of oncologic ${ }^{18} \mathrm{~F}$ FDG PET/CT compared with other tumor types due to its inherent biologic predilection for fatty acid rather than glucose metabolism. Because of this unmet clinical need, there has been an increased interest and development of emerging non- ${ }^{18}$ F-FDG PET radiotracers for prostate cancer including choline $\left({ }^{11} \mathrm{C}\right.$-choline, ${ }^{18} \mathrm{~F}$-fluorocholine, ${ }^{18} \mathrm{~F}$-fluoromethylcholine), acetate $\left({ }^{11} \mathrm{C}\right.$-acetate, ${ }^{18} \mathrm{~F}$-fluoroacetate), and amino acid-based $\left({ }^{18} \mathrm{~F}\right.$-fluciclovine) agents. Some of these radiotracers have successfully received U.S. Food and Drug Administration New Drug Application approval for clinical use $\left({ }^{11} \mathrm{C}\right.$-choline, ${ }^{18} \mathrm{~F}$-fluciclovine $)$, which has been well received. However, recent developments and promising initial clinical studies with novel ${ }^{18} \mathrm{~F}$ - and ${ }^{68} \mathrm{Ga}$-labeled low-molecularweight prostate-specific membrane antigen (PSMA) PET radiotracers have generated increased excitement because of their very promising early results for improved detection and staging of prostate cancer beyond conventional imaging modalities and even other non- ${ }^{18}$ F-FDG PET radiotracers $(2,3)$. However, these new PSMA PET radiotracers will need to deliver on this initial promise in the next phase of their development for it to become a valuable tool for patient management and drug development for prostate cancer in forthcoming systematic multicenter clinical trials. The authors of 2 new proposed PSMA PET criteria to facilitate prostate cancer reporting and classification make important and needed first steps in the next phase of development of this class of molecular imaging agents for prostate cancer, also applicable to other non-PSMA PET agents $(4,5)$.

PSMA is a viable target for prostate cancer imaging because of its high expression on most prostate cancer cells and association with more aggressive prostate cancer biology (3). The clinical relevance of PSMA PET for prostate cancer imaging includes a high signal-to-background signal for improved tumor detection, especially but not limited to, localization of the sites of biochemical

Received Nov. 23, 2017; revision accepted Dec. 14, 2017.

For correspondence or reprints contact: Steve Y. Cho, University of Wisconsin School of Medicine and Public Health, WIMR1, Rm. 7139, 1111 Highland Ave., Madison, WI 53705.

E-mail: scho@uwhealth.org

Published online Jan. 4, 2018

COPYRIGHT (c) 2018 by the Society of Nuclear Medicine and Molecular Imaging. DOI: 10.2967/jnumed.117.204057 recurrence compared with conventional imaging and even to choline PET at low serum prostate-specific antigen levels (6,7). Despite this high performance, false-positive lesions have been reported and these pitfalls, including PSMA PET uptake in normal physiologic distribution (e.g., ureter, sympathetic chain ganglion), inflammatory processes (e.g., sarcoidosis), benign osseous processes (e.g., Paget's disease), and tumor neovasculature of nonprostate malignancies (e.g., renal cell carcinoma), need to be taken into consideration in future criteria (8).

A standardized reporting system for incorporation of PSMA PET imaging to meet upcoming clinical diagnostic and clinical research needs requires an efficient but accurate method to meet the needs for the imager, referring treating clinicians, and multicenter clinical trials. These clinical and research needs, while overlapping, have unique requirements. Clinical diagnostic reporting tools need to be simple, efficient, and clinical action-directed, while adaptable to unique clinical situations. Incorporation of PSMA PET findings into current anatomic imaging-based diagnosis staging criteria is also needed to efficiently communicate these findings to support clinician therapeutic management decisions. Research reporting tools need to be reproducible and accurately allow for stratification of patient cohorts as well as provide the structure for pooling of patient data for multicenter trials. Both reporting tools need to be adaptable to allow for incorporation of updates and changes as we learn more about PSMA PET imaging with new research findings and clinical experience. The Reporting and Data System (RADS) is a quality assurance tool applied to pretherapy initial diagnosis of primary prostate cancer. The tumor, node, and metastasis (TNM) classification of malignant tumors is a notation system that describes the stage and anatomic extent of a solid tumor. Both PSMA-RADS and PROMISE classifications propose incorporation of PSMA PET into existing systems of tumor diagnosis and staging classifications, albeit with complementary proposals based on the RADS and TNM classification, respectively.

PSMA-RADS version 1.0 proposes a standardized method to "allow for an accurate and efficient means of relaying findings to referring providers" and "facilitate the collection of data for large prospective trials" (4). It proposes reporting an imager's level of certainty regarding PSMA PET findings using a 5-point scale (ranging from PSMA-RADS-1, benign; PSMA-RADS-2, likely benign; PSMA-RADS-3, equivocal; PSMA-RADS-4, prostate cancer highly likely; PSMA-RADS-5, prostate cancer almost certainly present), with additional sublevels for each category. PSMA-RADS is proposed for categorization of findings outside the prostate in pelvic or distant metastatic disease and does not address primary prostate cancer. An individual lesion- and patient-based PSMA-RADS report is also proposed, an important perspective requiring simplification of complex imaging findings in patients with widespread metastatic disease. A strength of this proposed method is the incorporation of clearly defined levels of confidence in imaging 
findings with actionable recommendations, ranging from benign, equivocal (requiring confirmatory workup or follow-up imaging), or positive disease not requiring confirmatory biopsy. These criteria also importantly add required reporting guidelines (e.g., PSA level, date of last treatment, dose of radiotracer injection) and address the oligometastatic disease setting (state of disease with $\leq 5$ metastatic sites), proposing a PSMA-RADS score for each site of suspected metastasis given emerging focal ablative therapy options for individual sites of metastasis in this disease setting. However, the interpretation of PET imaging findings in PSMA-RADS termed "typical" or "atypical" for prostate cancer is an issue that will require clarification and further refinement with more research studies and development of PSMA PET interpretation guidelines.

PROMISE incorporates PSMA PET-based molecular imaging (although the authors propose that these criteria can also be used for non-PSMA radiotracers) also with the goal of adopting a "unified language...for organizing findings in comprehensible categories" to allow for efficient communication of findings among "physicians and institutions" (5). Their proposal provides a first step for a molecular imaging-based TNM) reporting system (miTNM version 1.0) for PSMA PET/CT and PET/MR. An important aspect of these criteria incorporates PSMA PET findings with current anatomic imaging-based clinical reporting criteria to report the location and extent of sites of disease including primary prostate (adopting and layering in Prostate Imaging Reporting and Data System [PI-RADS] for primary prostate cancer), pelvic, and extrapelvic sites of tumor spread. A uniform adoption of this anatomic localization system using the same locations and terminology agreed on among both the imager and the treating clinician is critical for effective communication as well as comparing results across clinical studies, especially for studies involving pathologic lymph node dissection and radiation therapy planning. This proposal also incorporates a diagnostic confidence score based on incorporation of relevant clinical and conventional anatomic imaging. A unique contribution of these criteria is primary prostate cancer reporting with a proposed 6-segment (sextant) schema for use with PSMA PET/MR. An issue is that future clinical applications may need more localized anatomic definition (from 6 to 12 segments incorporating anterior/posterior segments) or to adopt use of a MRI sector segmentation map used in PI-RADS version 2 for MRI (39 sectors/regions: 36 for the prostate, 2 for the seminal vesicles, and 1 for the external urethral sphincter). Another important issue that will need further clarification and discussion is the anatomic definition of pelvic and extrapelvic region among imagers, radiation oncologists, and surgeons as this anatomic boundary will be critical in correlating imaging findings with surgical nodal dissection or pelvic radiation fields.

Visual criteria to define whether there is PSMA PET-positive or -negative findings for prostate cancer was proposed in PROMISE but not discussed in PSMA-RADS. PROMISE proposes the miPSMA score using internal organ reference PET uptake. This is similar to the visual PET classification criteria incorporated in both clinical and research settings by the current lymphoma Lugano criteria using a 5 -point ${ }^{18} \mathrm{~F}$-FDG PET Deauville visual scale with normal liver and mediastinal blood pool as reference regions (9). The proposed miPSMA criteria uses blood pool, liver, and parotid gland for reference regions (with spleen replacing liver in radiotracers with liver dominant excretion PET agents such as ${ }^{18}$ F-PSMA 1007), with a miPSMA score of 0 ( $<$ blood pool), 1 ( $\geq \mathrm{BP},<$ liver), 2 ( $\geq$ liver, $<$ parotid gland), and 3 ( $\geq$ parotid gland). However, the use of this proposed
miPSMA score would need adapting and verification, with some concern that PSMA uptake in these reference regions may vary among patients and different PSMA PET radiotracers, resulting in variability of reporting and poor reproducibility. There is also concern that certain lesions, especially small subcentimeter lymph nodes at sites of tumor recurrence, may be graded an miPSMA score of 0 but when read very sensitively any visually discernible activity may be considered metastatic disease, depending on our threshold for sensitivity in our interpretation needed for certain clinical settings such as biochemical recurrence with low serum PSA levels.

Important issues relevant to future versions of PSMA PET criteria but too premature to be addressed at this time with available scientific data including the use of a semiquantitative SUV-based PSMA PET metrics for prostate cancer detection and response assessment. The effect of androgen therapy and its modulation and effect on PSMA expression and therefore the PSMA PET signal in a castration-sensitive or castration-resistant disease setting will also need to be addressed in future criteria (10).

Prospective multicenter studies with PSMA PET can be best served by incorporating the important complementary information proposed in PSMA-RAD (Table 1 from PSMA-RADS) and PROMISE (Fig. 2 from PROMISE) in a combined reporting system. PROMISE addresses the anatomic regional definition of primary disease and recurrence, whereas PSMA-RADS delineates an imager's level of certainty regarding PSMA PET findings for metastatic disease. A multidisciplinary PSMA PET working group, similar to the international working group for lymphoma, could help better consolidate and update PSMA PET imaging criteria based on available scientific data and adapt to the changing landscape and needs of prostate cancer therapies. Future trials can prospectively test and validate these proposed criteria in initial studies by assessing the predictive value for detection of primary and metastatic disease with reference histopathology correlation, clinical outcome in PSMA-directed ablative therapies, and change in clinical management. Standardized criteria can also be adapted to compare PSMA PET with other emerging imaging modalities, including novel PET and SPECT imaging agents, to select the best modality for particular prostate cancer clinical scenarios. We cannot lose this momentum and opportunity presented to us in the molecular imaging community to lay the proper groundwork to deliver on the promise of PSMA PET molecular imaging for the sake of our current and future patients afflicted with prostate cancer.

\section{REFERENCES}

1. Kairos. Wikipedia website. https://en.wikipedia.org/wiki/Kairos. Accessed January 16,2018

2. Eiber M, Fendler WP, Rowe SP, et al. Prostate-specific membrane antigen ligands for imaging and therapy. J Nucl Med. 2017;58(suppl 2):67S-76S

3. Rowe SP, Gorin MA, Allaf ME, et al. PET imaging of prostate-specific membrane antigen in prostate cancer: current state of the art and future challenges. Prostate Cancer Prostatic Dis. 2016;19:223-230.

4. Rowe SP, Pienta KJ, Pomper MG, Gorin MA. Proposal for a structured reporting system for prostate-specific membrane antigen-targeted PET imaging: PSMARADS Version 1.0. J Nucl Med. 2018;59:479-485.

5. Eiber M, Herrmann K, Calais J, et al. Prostate cancer Molecular Imaging Standardized Evaluation (PROMISE): proposed miTNM classification for the interpretation of PSMA-ligand PET/CT. J Nucl Med. 2018:469-478.

6. Perera M, Papa N, Christidis D, et al. Sensitivity, specificity, and predictors of positive ${ }^{68} \mathrm{Ga}$-prostate-specific membrane antigen positron emission tomography in advanced prostate cancer: a systematic review and meta-analysis. Eur Urol. 2016;70:926-937.

7. von Eyben FE, Picchio M, von Eyben R, Rhee H, Bauman G. ${ }^{68}$ Ga-labeled prostate-specific membrane antigen ligand positron emission tomography/ computed tomography for prostate cancer: a systematic review and meta-analysis. Eur Urol Focus. 2016;S2405-4569(16)30160-2. 
8. Sheikhbahaei S, Afshar-Oromieh A, Eiber M, et al. Pearls and pitfalls in clinical interpretation of prostate-specific membrane antigen (PSMA)-targeted PET imaging. Eur J Nucl Med Mol Imaging. 2017;44:2117-2136.

9. Barrington SF, Mikhaeel NG, Kostakoglu L, et al. Role of imaging in the staging and response assessment of lymphoma: consensus of the International Conference on Malignant Lymphomas Imaging Working Group. J Clin Oncol. 2014;32: 3048-3058.

10. Evans MJ, Smith-Jones PM, Wongvipat J, et al. Noninvasive measurement of androgen receptor signaling with a positron-emitting radiopharmaceutical that targets prostatespecific membrane antigen. Proc Natl Acad Sci USA. 2011;108:9578-9582.

\section{Erratum}

In the article "Quantification of Task-Specific Glucose Metabolism with Constant Infusion of ${ }^{18}$ F-FDG" by Hahn et al. (J Nucl Med. 2016;57:1933-1940), the task-specific glucose metabolism as defined in Equations 6 and 7 represents an oversimplification. Considering that the slopes of the baseline and task regressors are different, the model was refined as follows:

Task-specific changes in metabolism are proportional to changes in the slope of the time-activity curve, hence, changing baseline metabolism by $\beta_{\text {task }} \times$ regressor ${ }_{\text {task }}$

$$
K_{i_{\text {_total }}} \propto \beta_{\text {base }} \times \text { regressor }_{\text {base }}+\beta_{\text {task }} \times \text { regressor }_{\text {task }}
$$

from which follows that task-specific metabolism is proportional to

$$
K_{i_{-} \text {task }} \propto \beta_{\text {task }} \times \text { regressor }_{\text {task }}
$$

For the quantification with the Patlak plot, the task regressor was then reconstructed as a linear function with the same slope as defined in the GLM analysis. This assumes that task-related glucose metabolism is constant throughout the task.

As a consequence, the task-specific values in CMRGlu and percentage signal changes are higher by a factor of approximately 4 , as shown in the table below. Since this factor slightly varies across subjects and regions, the statistics were adapted but the interpretations remained stable. The authors regret the error.

TABLE 1

\begin{tabular}{|c|c|c|c|c|c|c|c|}
\hline Region & $\mathrm{x}(\mathrm{mm})$ & $\mathrm{y}(\mathrm{mm})$ & $\mathrm{z}(\mathrm{mm})$ & t-value & CMRGlu BL ( $\mu \mathrm{mol} / 100 \mathrm{~g} / \mathrm{min})$ & CMRGlu task ( $\mu \mathrm{mol} / 100 \mathrm{~g} / \mathrm{min})$ & Signal change (\%) \\
\hline \multicolumn{8}{|l|}{ Eyes open $>$ baseline } \\
\hline Lingual $\mathrm{L}$ & -6 & -88 & -14 & $9.4^{*}$ & $26.2 \pm 4.1$ & $3.3 \pm 1.4$ & $12.9 \pm 5.4$ \\
\hline Intracalcarine $L$ & -8 & -84 & 8 & $9.7^{\star}$ & $25.7 \pm 4.2$ & $2.8 \pm 1.1$ & $11.0 \pm 4.1$ \\
\hline Supracalcarine $\mathrm{R}$ & 16 & -66 & 14 & $11.0^{*}$ & $28.3 \pm 4.6$ & $2.3 \pm 0.8$ & $8.1 \pm 3.2$ \\
\hline Occ Pole R & 8 & -90 & 32 & $10.2^{*}$ & $17.7 \pm 3.4$ & $3.8 \pm 1.5$ & $21.2 \pm 6.9$ \\
\hline Cuneus & 0 & -82 & 26 & $11.2^{\star}$ & $27.6 \pm 4.2$ & $2.3 \pm 0.7$ & $8.4 \pm 2.3$ \\
\hline Cereb Crus I L & -46 & -60 & -34 & $11.4^{\star}$ & $23.0 \pm 3.5$ & $3.4 \pm 1.2$ & $14.7 \pm 4.6$ \\
\hline Cereb Crus I R & 54 & -52 & -34 & $12.4^{\star}$ & $11.5 \pm 2.4$ & $2.6 \pm 0.8$ & $23.6 \pm 7.5$ \\
\hline Cereb Crus II L & -12 & -88 & -26 & $13.5^{\star}$ & $19.7 \pm 3.5$ & $4.4 \pm 1.3$ & $22.8 \pm 7.1$ \\
\hline Cereb Crus II R & 4 & -82 & -26 & $9.1^{*}$ & $16.6 \pm 2.9$ & $4.3 \pm 1.8$ & $25.6 \pm 9.5$ \\
\hline Cereb VI L & -26 & -54 & -26 & $12.4^{*}$ & $22.6 \pm 3.0$ & $2.7 \pm 0.8$ & $12.2 \pm 4.6$ \\
\hline Cereb Vermis crus II & -2 & -80 & -30 & $11.2^{*}$ & $18.8 \pm 3.1$ & $3.5 \pm 1.2$ & $18.7 \pm 6.4$ \\
\hline Cereb Vermis VIIla & -8 & -66 & -40 & $10.8^{*}$ & $20.7 \pm 3.0$ & $2.6 \pm 0.9$ & $12.7 \pm 5.4$ \\
\hline Hippocampus L & -30 & -18 & -12 & $10.2^{\star}$ & $16.3 \pm 2.4$ & $3.1 \pm 1.2$ & $19.6 \pm 8.8$ \\
\hline \multicolumn{8}{|l|}{ Eyes open < baseline } \\
\hline Frontal superior $\mathrm{L}$ & -18 & 32 & 44 & $-7.5^{\#}$ & $26.7 \pm 5.1$ & $-1.9 \pm 1.0$ & $-7.0 \pm 2.9$ \\
\hline Postcentral L & -40 & -24 & 58 & -0.8 & $27.6 \pm 4.3$ & $-0.5 \pm 2.3$ & $-1.4 \pm 7.9$ \\
\hline \multicolumn{8}{|c|}{ Finger tapping $>$ baseline } \\
\hline Postcentral L & -40 & -24 & 58 & $12.5^{\star}$ & $27.6 \pm 4.3$ & $6.3 \pm 2.6$ & $22.8 \pm 9.6$ \\
\hline Precentral L & -32 & -16 & 70 & $11.0^{*}$ & $19.0 \pm 3.5$ & $3.9 \pm 1.7$ & $20.2 \pm 7.8$ \\
\hline Cereb VI L & -32 & -56 & -26 & $16.6^{*}$ & $24.8 \pm 3.5$ & $3.9 \pm 1.0$ & $15.9 \pm 5.0$ \\
\hline Cereb VIIla L & -34 & -60 & -56 & $13.1^{*}$ & $21.8 \pm 3.0$ & $4.7 \pm 1.4$ & $21.4 \pm 5.6$ \\
\hline Cereb VIIla R & 28 & -64 & -54 & $11.4^{*}$ & $23.1 \pm 3.1$ & $3.7 \pm 2.2$ & $17.0 \pm 12.2$ \\
\hline Cereb VIIII R & 10 & -64 & -52 & $11.8^{\star}$ & $20.7 \pm 2.7$ & $3.8 \pm 1.2$ & $18.4 \pm 6.2$ \\
\hline Hippocampus L & -28 & -16 & -12 & $10.6^{*}$ & $16.3 \pm 2.5$ & $3.4 \pm 1.3$ & $21.1 \pm 7.8$ \\
\hline \multicolumn{8}{|c|}{ Finger tapping < baseline } \\
\hline Intracalcarine L & -8 & -84 & 8 & -0.9 & $25.7 \pm 4.2$ & $-0.3 \pm 2.2$ & $-0.4 \pm 8.7$ \\
\hline
\end{tabular}

Task-Specific Changes in Glucose Metabolism for Eyes-Open Condition and Right-Finger Tapping as Compared with Baseline 